\title{
The Reduced Order Method for Solving the Linear Complementarity Problem with an M-Matrix
}

\author{
Xi-Ming Fang ${ }^{1} \cdot$ Heng-Jun $\mathrm{Zhao}^{2} \cdot$ Jing $\mathrm{Li}^{3} \cdot$ Zhijun Qiao $^{4}$
}

Received: 6 November 2021 / Accepted: 12 January 2022 / Published online: 31 January 2022

(c) The Author(s) 2022, corrected publication 2022

\begin{abstract}
In this paper, by seeking the zero and the positive entry positions of the solution, we provide a direct method, called the reduced order method, for solving the linear complementarity problem with an $M$-matrix. By this method, the linear complementarity problem is transformed into a low order linear complementarity problem with some low order linear equations and the solution is constructed by the solution of the low order linear complementarity problem and the solutions of these low order linear equations in the transformations. In order to show the accuracy and the effectiveness of the method, the corresponding numerical experiments are performed.
\end{abstract}

Keywords Linear complementarity problem $\cdot M$-matrix $\cdot$ Solution $\cdot$ Direct method

\section{Introduction}

The linear complementarity problem is to find a real vector $x \in R^{n}$ satisfying

\author{
Zhijun Qiao \\ zhijun.qiao@utrgv.edu \\ Xi-Ming Fang \\ fangxm504@163.com
}

Heng-Jun Zhao

zhhj.zhao@139.com

Jing Li

leejing@bjut.edu.cn

1 School of Mathematics and Statistics, Zhaoqing University, Zhaoqing 526000, People's Republic of China

2 Department of Mathematics and Finance, Key Laboratory of Graph Theories and Applications, Chongqing University of Arts and Sciences, Chongqing 400000, People's Republic of China

3 Interdisciplinary Research Institute, Faculty of Science Beijing University of Technology, Beijing 100124, People's Republic of China

4 School of Mathematical and Statistical Sciences, The University of Texas Rio Grande Valley, Edinburg 78539, Texas, USA 


$$
x^{\mathrm{T}} w=0, x \geq 0, w=A x+q \geq 0,
$$

where $A \in R^{n \times n}, q \in R^{n}$ and the superscript 'T' denotes the transpose of a vector. For our convenience, let us denote this problem as $\operatorname{LCP}(A, q)$. Many problems can be transformed into the form (1), for instance, the elastic contact problem, the linear and quadratic programming problems and the market equilibrium problems, see [1-6] and the references therein.

It is well-known that the $\operatorname{LCP}(A, q)$ has a unique solution for any $q \in R^{n}$ if and only if the system matrix $A$ is a $P$-matrix. The $P$-matrices include many types, for example, the positive definite matrix and the $H_{+}$-matrix, both of which have been investigated in both theory and the numerical algorithms aspects, see [1, 7-11] and the references therein. In recent decades, a series of solving methods for the $\operatorname{LCP}(A, q)$ are studied by many authors, such as the Lemke method [3], the principal pivot transformation method [12], the non-modulus linear method [13], the projected method [8], the chaotic iterative methods [14] and the modulus-based matrix splitting iteration methods [7], etc.. These solving approaches can be divided into two categories: the direct methods and the iteration methods. The first three methods mentioned above are the direct methods and the latter three methods are the iteration methods. Both the direct methods and the iteration methods have their advantages and both methods need some conditions. In general, when the $\operatorname{LCP}(A, q)$ has a small and dense system matrix, the direct methods are considered, and when the $\operatorname{LCP}(A, q)$ has a large and sparse system matrix, the iteration methods are considered. However, the criterion of applying the direct methods or the iteration methods is not unconditional, such as, if the sparse structure of $A$ can not be applied in the iteration methods but the direct methods are simple and effective, it is more better to consider the direct methods even if $A$ is a large matrix, since the solutions obtained by the direct methods are the exact solutions. For other materials connected with the solving methods, see [14-26] and the references therein. Besides the numerical algorithms for the $\operatorname{LCP}(A, q)$, the theoretical aspects have also been discussed, for instance, the existence and uniqueness of solution, the stability and sensitivity of the solution, the relationship between the $\operatorname{LCP}(A, q)$ and other problems, the error bound problems and the perturbation problems of the solution, see $[1,5,9,27-30]$ and other references.

In this paper, we study the direct method for solving the $\operatorname{LCP}(A, q)$ and consider a particular $\operatorname{LCP}(A, q)$ with the system matrix $A$ being an $M$-matrix. Based on the complementarity relation existed in the $\operatorname{LCP}(A, q)$ and the properties of the $M$-matrix, by seeking the zero and the positive entry positions of the solution, we transform the $\operatorname{LCP}(A, q)$ into an equivalent low order linear complementarity problem with a low order linear equations. Since the low order linear complementarity problem is established, then this transformation procession can be continued. In this way, we provide a reduced order method for solving the $\operatorname{LCP}(A, q)$ with an $M$-matrix. This method integrates the reducing order procession and the construction of the solution of the linear complementarity problem in together, and the numerical results show that the reduced order method is an effective method. 
The outline of this paper is as follows. We present the main results and the reduced order method in Sect. 2 and the pseudo codes of this method are given in Sect. 3.

\section{Main Results}

In this section, we consider the direct method for solving the $\operatorname{LCP}(A, q)$ with an $M$-matrix. Let us first give some definitions, notations and preliminary works. Most of them can be found in $[5,7,10]$. The real matrix $A=\left(a_{i j}\right)$ is denoted by $A \geq 0$ if $a_{i j} \geq 0, i, j=1,2, \ldots, n$. A matrix $A \in R^{n \times n}$ is called an $M$-matrix if it satisfies $A^{-1} \geq 0$ with $a_{i j} \leq 0(i \neq j), i, j=1,2, \ldots, n$. For an $M$-matrix, all of its principal submatrices are also $M$-matrices. The vector $v$ is denoted by $v \geq 0(>0)$ if $v_{i} \geq 0(>0)$ holds for $i=1,2, \ldots, n$.

Lemma 1 Let $A \in R^{n \times n}$ be an M-matrix, and suppose $x^{*}$ is the solution of the $\operatorname{LCP}(A, q)$, then

(i) if $q \leq 0, x^{*}=A^{-1}(-q)$;

(ii) if $q \geq 0, x^{*}=0$.

Proof Since the matrix $A$ is an $M$-matrix, so $A^{-1} \geq 0$ and the $\operatorname{LCP}(A, q)$ has a unique solution.

If $q \leq 0$, then $A^{-1}(-q) \geq 0$ and $A\left(A^{-1}(-q)\right)+q=(-q)+q=0$, that is $\left(A^{-1}(-q)\right)^{\mathrm{T}}\left[A\left(A^{-1}(-q)\right)+q\right]=0$, so $x^{*}=A^{-1}(-q)$.

If $q \geq 0$, then $0^{\mathrm{T}}(A 0+q)=0$, so $x^{*}=0$.

Therefore, the conclusion is proved.

Lemma 1 considers two particular cases of the $\operatorname{LCP}(A, q)$, in the following, we discuss the general case.

Theorem 1 Let $A \in R^{n \times n}$ be an M-matrix, and suppose $x^{*}$ is the solution of the $\operatorname{LCP}(A, q)$. Let $q=\left(q_{1}, q_{2}, \ldots, q_{n}\right)^{\mathrm{T}} \in R^{n}$. Denote $S_{N}=\left\{i: q_{i}<0\right\}=\left\{i_{1}, i_{2}, \ldots, i_{s}\right\} \neq \Phi$, $S_{P}=\left\{j: q_{j} \geq 0\right\}=\left\{j_{1}, j_{2}, \ldots, j_{t}\right\}, \quad \mathfrak{q}_{1}=\left(q_{i_{1}}, q_{i_{2}}, \ldots, q_{i_{s}}\right)^{\mathrm{T}}, \quad \mathfrak{q}_{2}=\left(q_{j_{1}}, q_{j_{2}}, \ldots, q_{j_{t}}\right)^{\mathrm{T}}$, $\hat{x}_{1}^{*}=\left(x_{i_{1}}^{*}, x_{i_{2}}^{*}, \ldots, x_{i_{s}}^{*}\right)^{\mathrm{T}}, \hat{x}_{2}^{*}=\left(x_{j_{1}}^{*}, x_{j_{2}}^{*}, \ldots, x_{j_{t}}^{*}\right)^{\mathrm{T}}$. Denote $A_{\left(S_{N}, S_{N}\right)}$ as the principal submatrix of $A$, and denote $A_{\left(S_{P}, S_{N}\right)}$ as the sub-matrix of $A$ with the row and column indices coming from the sets $S_{P}, S_{N}$, respectively, and $v=A_{\left(S_{N}, S_{N}\right)}^{-1}\left(-\mathfrak{q}_{1}\right)$. If $\mathfrak{q}_{2}+A_{\left(S_{P}, S_{N}\right)} v \geq 0$, then $\hat{x}_{1}^{*}=v, \hat{x}_{2}^{*}=0$.

Proof From the definition of $S_{N}$ and $S_{P}$, we know $s+t=n$. Since $A$ is an $M$-matrix, $A_{\left(S_{N}, S_{N}\right)}$ is an $M$-matrix too, therefore the vector $v=A_{\left(S_{N}, S_{N}\right)}^{-1}\left(-\mathfrak{q}_{1}\right)$ is a positive vector. If we denote the indices of the vector $v$ by $i_{1}, i_{2}, \ldots, i_{s}$ corresponding to the elements of set $S_{N}$, and set $x^{*}=\left(x_{1}^{*}, x_{1}^{*}, \ldots, x_{n}^{*}\right)^{\mathrm{T}}$, where $x_{i}^{*}=0$ if $i \in S_{P}$ and $x_{i}^{*}=x_{i_{k}}^{*}=v_{i_{k}}$ if 
$i \in S_{N}$, and set $w^{*}=\left(w_{1}^{*}, w_{2}^{*}, \ldots, w_{n}^{*}\right)^{\mathrm{T}}$, where $\quad w_{i}^{*}=0 \quad$ if $\quad i \in S_{N} \quad$ and $w_{i}^{*}=q_{i}+A_{\left(i, S_{N}\right)} v \geq 0$ if $i \in S_{P}$, then we have $x^{*} \geq 0, w^{*} \geq 0$ with

$$
\left(\begin{array}{ll}
I-A
\end{array}\right)\left(\begin{array}{c}
w^{*} \\
x^{*}
\end{array}\right)=q, \quad x^{* \mathrm{~T}} w^{*}=0 .
$$

Thus, $x^{*}$ is the solution of the $\operatorname{LCP}(A, q)$. From the constructions of $x^{*}, w^{*}$, and the unique property of the solution for the $\operatorname{LCP}(A, q)$ with an $M$-matrix, we know $\hat{x}_{1}^{*}=v, \hat{x}_{2}^{*}=0$, which constitute the whole solution $x^{*}$ of the $\operatorname{LCP}(A, q)$. So the conclusion is established.

From Theorem 1 and the proof, we can find that the solution of the $\operatorname{LCP}(A, q)$ is composed of two parts in some conditions: one is the zero entries part, and the other one is the positive entries part which can be obtained by solving a low order linear equations. If the conditions are not satisfied, we have the following result about the solution.

Theorem 2 Let $A \in R^{n \times n}$ be an M-matrix, and suppose $x^{*}$ is the solution of the $\operatorname{LCP}(A, q)$. Let $q=\left(q_{1}, q_{2}, \ldots, q_{n}\right)^{\mathrm{T}} \in R^{n}$ and $w^{*}=A x^{*}+q=\left(w_{1}^{*}, w_{2}^{*}, \ldots, w_{n}^{*}\right)^{\mathrm{T}} \in R^{n}$. Denote $S_{N}=\left\{i: q_{i}<0\right\}=\left\{i_{1}, i_{2}, \ldots, i_{s}\right\} \neq \Phi, \quad S_{P}=\left\{j: q_{j} \geq 0\right\}=\left\{j_{1}, j_{2}, \ldots, j_{t}\right\}$, $\mathfrak{q}_{1}=\left(q_{i_{1}}, q_{i_{2}}, \ldots, q_{i_{s}}\right)^{\mathrm{T}}, \mathfrak{q}_{2}=\left(q_{j_{1}}, q_{j_{2}}, \ldots, q_{j_{t}}\right)^{\mathrm{T}}, \hat{x}_{1}^{*}=\left(x_{i_{1}}^{*}, x_{i_{2}}^{*}, \ldots, x_{i_{s}}^{*}\right)^{\mathrm{T}}, \hat{x}_{2}^{*}=\left(x_{j_{1}}^{*}, x_{j_{2}}^{*}, \ldots, x_{j_{t}}^{*}\right)^{\mathrm{T}}$, $\hat{w}_{1}^{*}=\left(w_{i_{1}}^{*}, w_{i_{2}}^{*}, \ldots, w_{i_{s}}^{*}\right)^{\mathrm{T}}$ and $\hat{w}_{2}^{*}=\left(w_{j_{1}}^{*}, w_{j_{2}}^{*}, \ldots, w_{j_{t}}^{*}\right)^{\mathrm{T}}$. Denote $A_{\left(S_{N}, S_{N}\right)}, A_{\left(S_{P}, S_{P}\right)}, A_{\left(S_{P}, S_{N}\right)}$, $A_{\left(S_{N}, S_{p}\right)}$ as the sub-matrices of $A$ with the row and column indices coming from the sets $S_{P}, S_{N}$, respectively, and $v=A_{\left(S_{N}, S_{N}\right)}^{-1}\left(-\mathfrak{q}_{1}\right)$. If $\mathfrak{q}_{2}+A_{\left(S_{P}, S_{N}\right)} v$ has negative entries, then the $\operatorname{LCP}(A, q)$ can be transformed into a low order equivalent linear complementarity problem $\operatorname{LCP}\left(A^{1}, q^{1}\right)$ with a linear system $N^{1} x=b^{1}$, where $A^{1}=A_{\left(S_{P}, S_{P}\right)}$ $-A_{\left(S_{P}, S_{N}\right)} A_{\left(S_{N}, S_{N}\right)}^{-1} A_{\left(S_{N}, S_{P}\right)}, q^{1}=\mathfrak{q}_{2}+A_{\left(S_{P}, S_{N}\right)} v, N^{1}=A_{\left(S_{N}, S_{N}\right)}, b^{1}=-\mathfrak{q}_{1}-A_{\left(S_{N}, S_{P}\right)} \hat{x}_{2}^{*}$ and $\hat{x}_{2}^{*}$ is the solution of the $\operatorname{LCP}\left(A^{1}, q^{1}\right)$.

Proof For

$$
A_{\left(S_{N}, S_{N}\right)} \hat{x}_{1}+A_{\left(S_{N}, S_{P}\right)} \hat{x}_{2}+\mathfrak{q}_{1}=\hat{w}_{1},
$$

and the matrix $A$ beng an $M$-matrix, $q_{i}<0$ for $i \in S_{N}$, and $\hat{w}_{1} \geq 0$, we know that for each $i \in S_{N}, x_{i}^{*} \neq 0$ holds. Thus $\hat{w}_{1}=0$, that is $\hat{w}_{1}^{*}=0$, then the part $\hat{x}_{1}^{*}$ of the solution $x^{*}$ should satisfy

$$
A_{\left(S_{N}, S_{N}\right)} \hat{x}_{1}^{*}=-\mathfrak{q}_{1}-A_{\left(S_{N}, S_{P}\right)} \hat{x}_{2}
$$

Solving this linear system equations for $\hat{x}_{1}^{*}$, we obtain

$$
\hat{x}_{1}^{*}=-A_{\left(S_{N}, S_{N}\right)}^{-1}\left[\mathfrak{q}_{1}+A_{\left(S_{N}, S_{P}\right)} \hat{x}_{2}\right] .
$$

Then by substitution, the other part $\hat{x}_{2}^{*}$ of the solution $x^{*}$ satisfies the equation

$$
\hat{w}_{2}-\left[A_{\left(S_{P}, S_{P}\right)}-A_{\left(S_{P}, S_{N}\right)} A_{\left(S_{N}, S_{N}\right)}^{-1} A_{\left(S_{N}, S_{P}\right)}\right] \hat{x}_{2}=\mathfrak{q}_{2}-A_{\left(S_{P}, S_{N}\right)} A_{\left(S_{N}, S_{N}\right)}^{-1} \mathfrak{q}_{1} .
$$


That is, the other part $\hat{x}_{2}^{*}$ of the solution $x^{*}$ satisfies the $\operatorname{LCP}\left(A^{1}, q^{1}\right)$

$$
\hat{x}_{2}^{\mathrm{T}} \hat{w}_{2}=0, \hat{w}_{2}=A^{1} \hat{x}_{2}+q^{1} \text {. }
$$

where the matrix

$$
A^{1}=A_{\left(S_{P}, S_{P}\right)}-A_{\left(S_{P}, S_{N}\right)} A_{\left(S_{N}, S_{N}\right)}^{-1} A_{\left(S_{N}, S_{P}\right)}
$$

is an $M$-matrix, which is the Schur complement of the principal sub-matrix $A\left(S_{N}, S_{N}\right)$, and

$$
q^{1}=\mathfrak{q}_{2}-A_{\left(S_{P}, S_{N}\right)} A_{\left(S_{N}, S_{N}\right)}^{-1} \mathfrak{q}_{1}=\mathfrak{q}_{2}+A_{\left(S_{P}, S_{N}\right)} v,
$$

which has the negative entries from the assumption of the theorem. Set the linear system (2) about $\hat{x}_{1}^{*}$ to be $N^{1} x=b^{1}$, if we solve (4) for $\hat{x}_{2}^{*}$, then from (2), (3) and (4), the conclusion of this theorem is proved.

About Theorem 2, we can see that the linear complementarity problem (4) for $\hat{x}_{2}^{*}$ should be solved first, then $\hat{x}_{1}^{*}$ can be obtained from the linear system (2), and the solution $x^{*}$ can be constructed by $\hat{x}_{1}^{*}$ and $\hat{x}_{2}^{*}$ at last. Moreover, we can find that since the low order linear complementarity problem $\operatorname{LCP}\left(A^{1}, q^{1}\right)$ is established, the transformation process of the linear complementarity problem can be continued until the negative index set $S_{N}=\Phi$ or the nonnegative set $S_{P}=\Phi$ for a low order linear complementarity problem. In these series of transformations, the condition $S_{N} \neq \Phi$ should be measured in advance each time. Since the linear complementarity problem is changed and these accompanied systems of linear equations remain the same in each transformation, we only need to solve the last low order linear complementarity problem, followed by solving the forward linear system step by step, then the solution of the original $\operatorname{LCP}(A, q)$ can be constructed in the end. Summarize the above discussions, we obtain the following Theorem 3 followed by the reduced order method for the $\operatorname{LCP}(A, q)$ from Theorem 2.

Theorem 3 Let $A \in R^{n \times n}$ be an M-matrix, and suppose $x^{*}$ is the solution of the $\operatorname{LCP}(A, q)$. Let $q=\left(q_{1}, q_{2}, \ldots, q_{n}\right)^{\mathrm{T}} \in R^{n}$ and $w^{*}=A x^{*}+q=\left(w_{1}^{*}, w_{2}^{*}, \ldots, w_{n}^{*}\right)^{\mathrm{T}} \in R^{n}$. Denote $S_{N}=\left\{i: q_{i}<0\right\}=\left\{i_{1}, i_{2}, \ldots, i_{s}\right\} \neq \Phi, \quad S_{P}=\left\{j: q_{j} \geq 0\right\}=\left\{j_{1}, j_{2}, \ldots, j_{t}\right\}$, $\mathfrak{q}_{1}=\left(q_{i_{1}}, q_{i_{2}}, \ldots, q_{i_{s}}\right)^{\mathrm{T}}, \mathfrak{q}_{2}=\left(q_{j_{1}}, q_{j_{2}}, \ldots, q_{j_{t}}\right)^{\mathrm{T}}, \hat{x}_{1}^{*}=\left(x_{i_{1}}^{*}, x_{i_{2}}^{*}, \ldots, x_{i_{s}}^{*}\right)^{\mathrm{T}}, \hat{x}_{2}^{*}=\left(x_{j_{1}}^{*}, x_{j_{2}}^{*}, \ldots, x_{j_{t}}^{*}\right)^{\mathrm{T}}$, $\hat{w}_{1}^{*}=\left(w_{i_{1}}^{*}, w_{i_{2}}^{*}, \ldots, w_{i_{s}}^{*}\right)^{\mathrm{T}}$ and $\hat{w}_{2}^{*}=\left(w_{j_{1}}^{*}, w_{j_{2}}^{*}, \ldots, w_{j_{t}}^{*}\right)^{\mathrm{T}}$. Denote $A_{\left(S_{N}, S_{N}\right)}, A_{\left(S_{P}, S_{P}\right)}, A_{\left(S_{P}, S_{N}\right)}$, $A_{\left(S_{N}, S_{p}\right)}$ as the sub-matrices of $A$ with the row and column indices coming from the sets $S_{P}, S_{N}$, respectively, and $v=A_{\left(S_{N}, S_{N}\right)}^{-1}\left(-\mathfrak{q}_{1}\right)$. If $q^{1}=\mathfrak{q}_{2}+A_{\left(S_{P}, S_{N}\right)}$ v has negative entries, then the $\operatorname{LCP}(A, q)$ can be transformed into a low order equivalent linear complementarity problem $\operatorname{LCP}\left(A^{l}, q^{l}\right)$ with a series of linear equations:

$$
\begin{gathered}
N^{r} x=b^{r}, r=1,2, \ldots, l, \\
\operatorname{LCP}\left(A^{l}, q^{l}\right) .
\end{gathered}
$$

where $l(>1)$ is an integer. For $r=1, N^{1}, A^{1}, b^{1}, q^{1}$ are same with those in Theorem 2. For $r=2,3, \ldots, l$, we have $N^{r}=A_{\left(S_{N}^{r-1}, S_{N}^{r-1}\right)}^{r-1}, b^{r}=-\mathfrak{q}_{1}^{r-1}-A_{\left(S_{N}^{r-1}, S_{P}^{r-1}\right)}^{r-1} \hat{x}_{2}^{(r-1) *}$ with 


$$
\begin{aligned}
& S_{N}^{r-1}=\left\{i: q_{i}^{r-1}<0\right\}=\left\{i_{1^{r-1}}, i_{2^{r-1}}, \ldots, i_{s^{r-1}}\right\} \neq \Phi, \\
& S_{P}^{r-1}=\left\{j: q_{j}^{r-1} \geq 0\right\}=\left\{j_{1^{r-1}}, i_{2^{r-1}}, \ldots, j_{t^{r-1}}\right\} .
\end{aligned}
$$

$x^{(r-1) *}$ is the solution of the $\operatorname{LCP}\left(A^{r-1}, q^{r-1}\right)$ with

$$
\begin{aligned}
A^{r-1} & =A_{\left(S_{P}^{r-2}, S_{P}^{r-2}\right)}^{r-2}-A_{\left(S_{P}^{r-2}, S_{N}^{r-2}\right)}^{r-2} A_{\left(S_{N}^{r-2}, S_{N}^{r-2}\right)}^{r-1} A_{\left(S_{N}^{r-2}, S_{P}^{r-2}\right)}^{r-2}, \\
q^{r-1} & =\mathfrak{q}_{2}^{r-2}-A_{\left(S_{P}^{r-2}, S_{N}^{r-2}\right)}^{r-2} A_{\left(S_{N}^{r-2}, S_{N}^{r-2}\right)}^{r-1} \mathfrak{q}_{1}^{r-2}, \\
\mathfrak{q}_{1}^{r-1} & =\left(q_{i_{1^{r-1}}}^{r-1}, q_{i_{2} r-1}^{r-1}, \ldots, q_{i_{s^{r-1}}}^{r-1}\right)^{\mathrm{T}},
\end{aligned}
$$

which is composed of $\hat{x}_{1}^{(r-1) *}$ and $\hat{x}_{2}^{(r-1) *}$ with $\hat{x}_{1}^{(r-1) *}$ satisfying $N^{r-1} x=b^{r-1}$ and $\hat{x}_{2}^{(r-1) *}$ being the solution of the $\operatorname{LCP}\left(A^{r-2}, q^{r-2}\right)$ for $r=3,4, \ldots, l$, and $\hat{x}_{2}^{(l) *}=0$ or $\hat{x}_{2}^{(l) *}=A^{l^{-1}}\left(-q^{l}\right)$.

According to Theorem 3, we obtain the reduced order method for solving the $\operatorname{LCP}(A, q)$ with an $M$-matrix, which is a direct method and the solution $x^{*}$ is obtained by means of construction from the solutions of some low order linear system equations and a low order linear complementarity problem. The linear complementarity problem is established in each transformation and the index sets that are connected with the sign of the constant vector in the linear complementarity problem need to be noted, which are applied to construct the solution $x^{*}$ at last. In addition, only when we determine the integer $l$, that is, the set $S_{N}^{l}$ which satisfies $S_{N}^{l}=\Phi$ or $S_{P}^{l}=\Phi$, then the process of solving the $\operatorname{LCP}(A, q)$ begins. At this time, we obtain the matrices $A^{l}, N^{l}$ and the solution $x^{(l) *}=0$ or $x^{(l) *}=A^{l^{-1}}\left(-q^{l}\right)$ of the $\operatorname{LCP}\left(A^{l}, q^{l}\right)$ from Lemma 1 . Solving $N^{l} x=b^{l}$ and implementing back substitution, then we begin the whole solving and construction process of $x^{*}$. The order of the linear complementarity problems is decreased gradually and it satisfies

$$
\sum_{r=1}^{l} \operatorname{Order}\left(N^{r} x=b^{r}\right)+\operatorname{Order}\left(\operatorname{LCP}\left(A^{l}, q^{l}\right)\right)=n
$$

and the solution $x^{*}$ is derived from construction, both of with are the main characters of the reduced order method, whose concrete pseudo codes are shown in Sect. 3. Besides, based on the proofs of Theorem 2 and Theorem 3, if we modify the reduced order method and add the expression of $w$, then $w^{*}$ can be obtained accompanied with the solution $x^{*}$, that is, $w^{*}$ can also be obtained by construction rather than by the expression $w^{*}=A x^{*}+q$. At the end of this paragraph, we remark here that the proposed reduced order method is different from [24] and [25], both of which did not reduce the order of the complementarity problem, however, the reduced order method integrates the searching process of the integer $l$ in Theorem 3, the solving processes of these low order linear equations and the construction of the solution $x^{*}$ in together. In addition, the former [24] discussed the $\operatorname{LCP}(A, q)$ with a Stieltjes matrix, which is a particular $M$-matrix, and the reduced order method can solve the $\operatorname{LCP}(A, q)$ with a general $M$-matrix. 


\section{Algorithm}

In this section, we present the pseudo codes of the reduced order method based on Lemma 1 and Theorems $1-3 .^{1}$

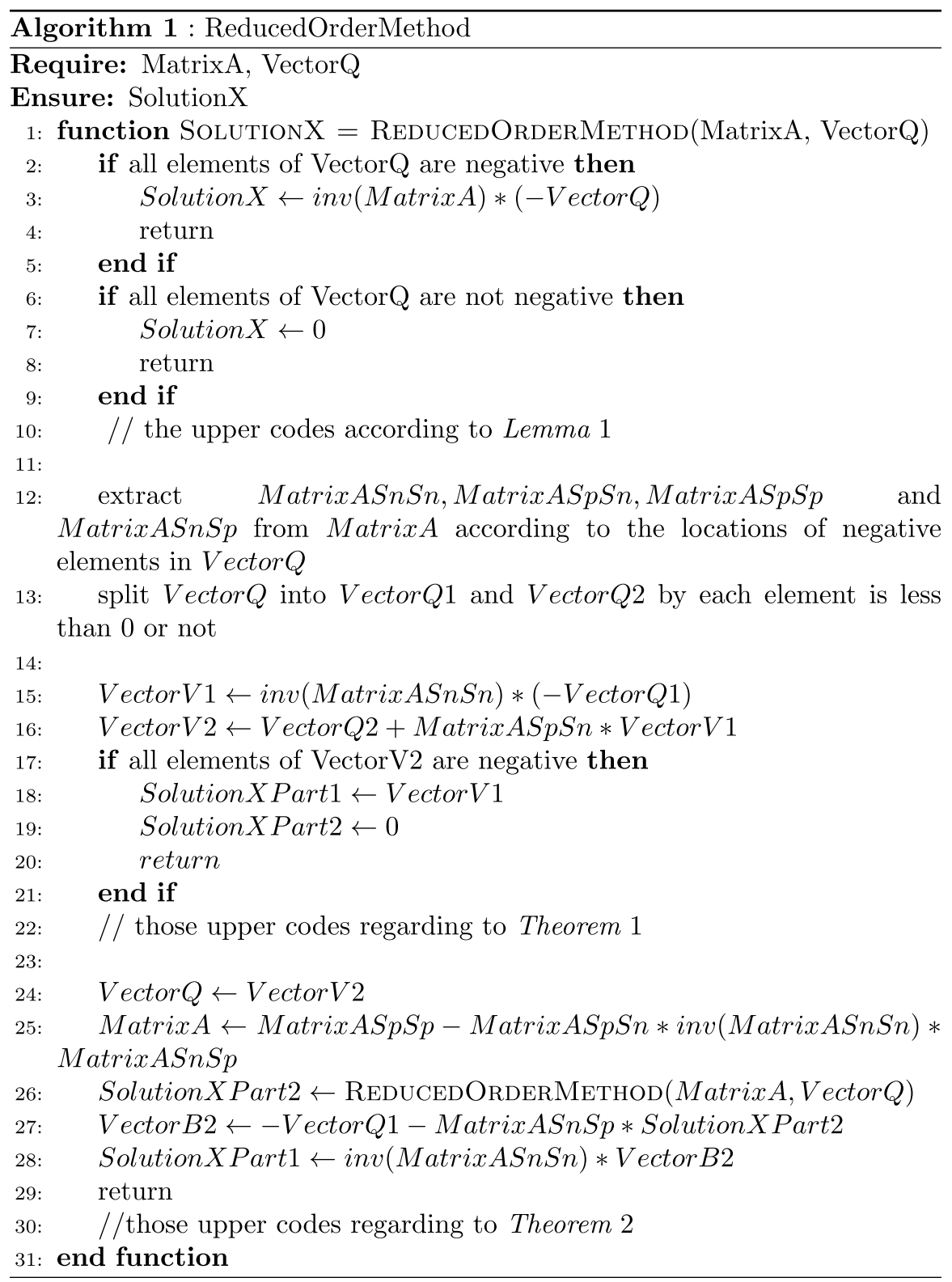

\footnotetext{
1 The matlab(2016a+) code is available on: https://github.com/HeanJean/reduceOrderMethhod.
} 


\section{Numerical Experiment}

In this section, we present three examples. The first example is a low order case, which is to show the solving process of the reduced order method. The second example is a high order case, which is to show the effectiveness of the reduced order method. The third example is to show that the reduced order method can be applied to solve the $\operatorname{LCP}(A, q)$ with an inverse $M$-matrix.

Example 1 Let the system matrix $A$ in the $\operatorname{LCP}(A, q)$ be

$$
A=\left(\begin{array}{ccccc}
1 & 0 & -1 & 0 & -1 \\
-1 & 2 & 0 & -1 & -1 \\
0 & -1 & 3 & -1 & 0 \\
-1 & 0 & -1 & 4 & -1 \\
0 & -1 & -1 & 0 & 5
\end{array}\right),
$$

and let the variable vector $q$ be

$$
\bar{q}=(-1,2,-1,2,1)^{\mathrm{T}}, \hat{q}=(-1,1,1,0,1)^{\mathrm{T}},
$$

respectively. It's easy to verify that the matrix $A$ is an $M$-matrix.

(I) For $\bar{q}$, we have the sets $S_{N}=\{1,3\} \neq \Phi, S_{P}=\{2,4,5\}$ and

$$
\begin{aligned}
& A_{\left(S_{N}, S_{N}\right)}=\left(\begin{array}{cc}
1 & -1 \\
0 & 3
\end{array}\right), \bar{v}=A_{\left(S_{N}, S_{N}\right)}^{-1}\left(-\overline{\mathfrak{q}}_{1}\right)=\left(\begin{array}{ll}
1 & \frac{1}{3} \\
0 & \frac{1}{3}
\end{array}\right)\left(\begin{array}{l}
1 \\
1
\end{array}\right)=\left(\begin{array}{c}
\frac{4}{3} \\
\frac{1}{3}
\end{array}\right) \text {. } \\
& \overline{\mathfrak{q}}_{2}+A_{\left(S_{P}, S_{N}\right)} \bar{v}=\left(\begin{array}{l}
2 \\
2 \\
1
\end{array}\right)+\left(\begin{array}{cc}
-1 & 0 \\
-1 & -1 \\
0 & -1
\end{array}\right)\left(\begin{array}{c}
\frac{4}{3} \\
\frac{1}{3}
\end{array}\right)=\left(\begin{array}{c}
\frac{2}{3} \\
\frac{1}{3} \\
\frac{2}{3}
\end{array}\right) \geq 0 .
\end{aligned}
$$

From Theorem 1, we know that the solution $\bar{x}^{*}$ is

$$
\bar{x}^{*}=\left(\frac{4}{3}, 0, \frac{1}{3}, 0,0\right)^{\mathrm{T}} \text {. }
$$

(II) For $\hat{q}$, we also have the sets $S_{N}=\{1,3\} \neq \Phi, S_{P}=\{2,4,5\}$ and

$$
\begin{aligned}
& \hat{v}=A_{\left(S_{N}, S_{N}\right)}^{-1}\left(-\hat{\mathfrak{q}}_{1}\right)=\bar{v}=\left(\begin{array}{c}
\frac{4}{3} \\
\frac{1}{3}
\end{array}\right), \\
& \hat{q}^{1}=\hat{\mathfrak{q}}_{2}+A_{\left(S_{P}, S_{N}\right)} \hat{v}=\left(\begin{array}{l}
1 \\
0 \\
1
\end{array}\right)+\left(\begin{array}{cc}
-1 & 0 \\
-1 & -1 \\
0 & -1
\end{array}\right)\left(\begin{array}{c}
\frac{4}{3} \\
\frac{1}{3}
\end{array}\right)=\left(\begin{array}{c}
-\frac{1}{3} \\
-\frac{5}{3} \\
\frac{2}{3}
\end{array}\right) .
\end{aligned}
$$


So, from Theorem 2, the $\operatorname{LCP}(A, \hat{q})$ can be transformed into a low order linear complementarity problem with a linear system of equations, that is,

$$
\begin{aligned}
\left(\begin{array}{cc}
1 & -1 \\
0 & 3
\end{array}\right)\left(\begin{array}{l}
x_{1} \\
x_{3}
\end{array}\right)=\left(\begin{array}{l}
1 \\
1
\end{array}\right)-\left(\begin{array}{ccc}
0 & 0 & -1 \\
-1 & -1 & 0
\end{array}\right)\left(\begin{array}{l}
\hat{x}_{2}^{*} \\
\hat{x}_{4}^{*} \\
\hat{x}_{5}^{*}
\end{array}\right), \\
\left(\begin{array}{l}
x_{2} \\
x_{4} \\
x_{5}
\end{array}\right)^{\mathrm{T}}\left(B\left(\begin{array}{l}
x_{2} \\
x_{4} \\
x_{5}
\end{array}\right)+\hat{q}^{1}\right)=0,
\end{aligned}
$$

where

$$
B=\left(\begin{array}{ccc}
2 & -1 & -1 \\
0 & 4 & -1 \\
-1 & 0 & 5
\end{array}\right)-\left(\begin{array}{cc}
-1 & 0 \\
-1 & -1 \\
0 & -1
\end{array}\right)\left(\begin{array}{cc}
1 & -1 \\
0 & 3
\end{array}\right)^{-1}\left(\begin{array}{ccc}
0 & 0 & -1 \\
-1 & -1 & 0
\end{array}\right)
$$

and the low order linear complementarity problem is established and can be simplified as

$$
\left(\begin{array}{l}
x_{2} \\
x_{4} \\
x_{5}
\end{array}\right)^{\mathrm{T}}\left(\left(\begin{array}{ccc}
\frac{5}{3} & -\frac{4}{3} & -2 \\
-\frac{2}{3} & \frac{10}{3} & -2 \\
-\frac{4}{3} & -\frac{1}{3} & 5
\end{array}\right)\left(\begin{array}{l}
x_{2} \\
x_{4} \\
x_{5}
\end{array}\right)+\left(\begin{array}{c}
-\frac{1}{3} \\
-\frac{5}{3} \\
\frac{2}{3}
\end{array}\right)\right)=0
$$

For the linear complementarity problem (6), we have $S_{N}^{1}=\{1,2\} \neq \Phi, S_{P}^{1}=\{3\}$ and

$$
\begin{aligned}
A_{\left(S_{N}^{1}, S_{N}^{1}\right)}^{1}= & \left(\begin{array}{cc}
\frac{5}{3} & -\frac{5}{3} \\
-\frac{2}{3} & \frac{10}{3}
\end{array}\right), \hat{v}^{1}=A_{\left(S_{N}^{1}, S_{N}^{1}\right)}^{1}{ }^{-1}\left(-\hat{\mathfrak{q}}_{1}^{1}\right)=\left(\begin{array}{cc}
\frac{5}{7} & \frac{2}{7} \\
\frac{1}{7} & \frac{5}{14}
\end{array}\right)\left(\begin{array}{c}
\frac{1}{3} \\
\frac{5}{3}
\end{array}\right)=\left(\begin{array}{c}
\frac{5}{7} \\
\frac{9}{14}
\end{array}\right), \\
& \hat{q}^{2}=\hat{\mathfrak{q}}_{2}^{1}+A_{\left(S_{P}^{1}, S_{N}^{1}\right)}^{1} \hat{v}^{1}=\frac{2}{3}+\left(-\frac{4}{3}-\frac{1}{3}\right)\left(\begin{array}{c}
\frac{5}{7} \\
\frac{9}{14}
\end{array}\right)=-\frac{1}{2} \leq 0 .
\end{aligned}
$$

So, from Theorem 3, the linear complementarity problem (6) can be transformed into a low order linear complementarity problem with a linear system again, that is,

$$
\begin{gathered}
\left(\begin{array}{cc}
\frac{5}{3} & -\frac{4}{3} \\
-\frac{2}{3} & \frac{10}{3}
\end{array}\right)\left(\begin{array}{l}
x_{2} \\
x_{4}
\end{array}\right)=\left(\begin{array}{c}
\frac{1}{3} \\
\frac{5}{3}
\end{array}\right)-\left(\begin{array}{l}
-2 \\
-2
\end{array}\right) \hat{x}_{5}^{*}, \\
x_{5} \mathrm{~T}\left(\left(5-\left(-\frac{4}{3}-\frac{1}{3}\right)\left(\begin{array}{cc}
\frac{5}{3} & -\frac{4}{3} \\
-\frac{2}{3} & \frac{10}{3}
\end{array}\right)^{-1}\left(\begin{array}{l}
-2 \\
-2
\end{array}\right)\right) x_{5}+\hat{q}^{2}\right)=0,
\end{gathered}
$$


where the second expression can be simplified as

$$
x_{5}^{\mathrm{T}}\left(2 x_{5}-\frac{1}{2}\right)=0 \text {. }
$$

Then, from Lemma 1 , the solution of (8) is $\hat{x}_{5}^{*}=\frac{\frac{1}{2}}{2}=\frac{1}{4}$. Combining with (7) and (5), the solution of the original $\operatorname{LCP}(A, \hat{q})$ can be constructed as

$$
\hat{x}^{*}=\left(\frac{16}{7}, \frac{17}{14}, \frac{29}{28}, \frac{25}{28}, \frac{1}{4}\right)^{\mathrm{T}} \text {. }
$$

From (II), we can find that the $\operatorname{LCP}(A, \hat{q})$ is transformed into a low order linear complementarity problem (8) with two low order linear systems of equations: (5) and (7).

Example 2 In this example, we consider the high order cases and show the effectiveness of the reduced order method, that is Algorithm 1 presented in Sect. 3. Let $A$ in the $\operatorname{LCP}(A, q)$ be a tridiagonal $M$-matrix, that is

$$
A=\operatorname{trid}(-1, d,-1) \in R^{n \times n}
$$

with $d=2, d=3$ and $d=4$, respectively. The matrix $A$ with $d=3$ arises from the American Option Pricing problems with $\tau=1$ in [23], see [6, 23] for details. The matrix $A$ with $d=4$ comes from the finite difference discretization of a free boundary value problem and we consider a particular case here, see [7, 17] for details. Set $q$ to be an arbitrary vector, that is $q$ is generated by

$$
q=\operatorname{randn}(n, 1)
$$

where "randn" is a function in Matlab software. We consider the running time (CPU), the number $(l)$ of the produced linear equations and the residual error (ERROR), where ERROR is defined as

$$
\operatorname{ERROR}=\operatorname{norm}(\min (x, A x+q)),
$$

here, "norm" and "min" are two functions in Matlab software; see [7, 17]. Set $n=1000$ and carry out 3 experiments for $d=2, d=3$ and $d=4$, respectively, then we obtain Table 1 as follows.

Here, we set $q_{i}(i=1,2,3)$ to be same for the three cases: $d=2, d=3$ and $d=4$. From Table 1, we can find that although the precision of the numerical solution will decease when too many linear equations are produced in the solving procession (the number $l$ of the linear equations is determined by the $\operatorname{LCP}(A, q)$ itself), the reduced order method is effective for solving the $\operatorname{LCP}(A, q)$ with an $M$-matrix.

Example 3 The reduced order method is presented for the $\operatorname{LCP}(A, q)$ with an $M$-matrix, however, if the inverse of $A$ is an $M$-matrix, that is, $A^{-1}$ is an $M$-matrix, then the $\operatorname{LCP}(A, q)$ can equivalently be reformulated as 


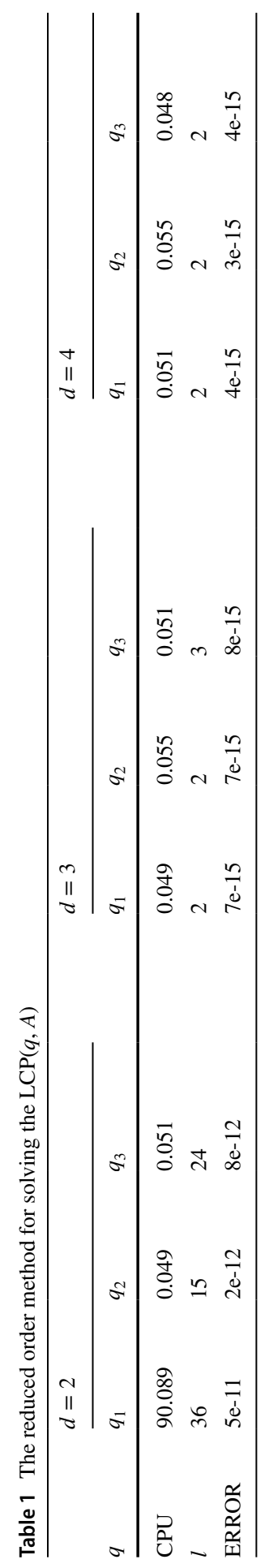




$$
w^{\mathrm{T}} x=0, w \geq 0, x=A^{-1} w+A^{-1}(-q) \geq 0,
$$

which is a linear complementarity problem with an $M$-matrix. If we denote the reformulated linear complementarity problem as $\operatorname{LCP}\left(A^{-1}, A^{-1}(-q)\right)$, then it can be solved for $w^{*}$ by the reduced order method. Thus we can obtain the solution $x^{*}$ of the $\operatorname{LCP}(A, q)$ easily through the expression

$$
x^{*}=A^{-1} w^{*}+A^{-1}(-q) .
$$

We consider a low order case to show this solving procession as follows. Set $A$ and $q$ in the $\operatorname{LCP}(A, q)$ to be

$$
A=\left(\begin{array}{ccccc}
3 & \frac{9}{7} & \frac{11}{7} & \frac{5}{7} & 1 \\
\frac{5}{2} & \frac{12}{7} & \frac{10}{7} & \frac{11}{14} & 1 \\
\frac{5}{4} & \frac{11}{14} & \frac{15}{14} & \frac{13}{28} & \frac{1}{2} \\
\frac{5}{4} & \frac{9}{14} & \frac{11}{14} & \frac{17}{28} & \frac{1}{2} \\
\frac{3}{4} & \frac{1}{2} & \frac{1}{2} & \frac{1}{4} & \frac{1}{2}
\end{array}\right), \bar{q}=(1,-3,2,-1,1)^{\mathrm{T}}, \hat{q}=(-1,0,-2,1,-1)^{\mathrm{T}},
$$

then $A$ is an inverse $M$-matrix. By the reduced order method and (9) with (10), the solutions are

$$
\bar{x}^{*}=\left(0, \frac{7}{4}, 0,0,0\right)^{\mathrm{T}}, \hat{x}^{*}=\left(0,0, \frac{7}{4}, 0, \frac{1}{4}\right)^{\mathrm{T}},
$$

respectively.

\section{Concluding Remark}

In this paper, we provide a direct method for solving the $\operatorname{LCP}(A, q)$ with an $M$-matrix, that is the reduced order method. By this method, the $\operatorname{LCP}(A, q)$ can ultimately be transformed into an equivalent complementarity problem with some low order linear equations. The numerical experiments show the accuracy and the effectiveness of this method.

Acknowledgements The authors are thankful to Professors Xiao-Dong Fan and Hong-Yin Shi for their fruitful discussions during Dr. Fang visits UTRGV.

Author Contributions All authors typed, read, reviewed, and approved the final manuscript.

Funding This work was partially supported by Zhaoqing University Doctoral Program (No. 611-612279), Chongqing Research Program of Basic Research and Frontier Technology (No. cstc2016jcyjA0554), 
National Natural Science Foundation of China (No. 11772007), Beijing Natural Science Foundation (No. Z180005), and High-level Foreign Experts Funding Program of Beijing (No. J202134).

Availability of Data and Material No data were used for this work.

\section{Declarations}

Conflict of Interest The authors have no conflicts of interest.

Open Access This article is licensed under a Creative Commons Attribution 4.0 International License, which permits use, sharing, adaptation, distribution and reproduction in any medium or format, as long as you give appropriate credit to the original author(s) and the source, provide a link to the Creative Commons licence, and indicate if changes were made. The images or other third party material in this article are included in the article's Creative Commons licence, unless indicated otherwise in a credit line to the material. If material is not included in the article's Creative Commons licence and your intended use is not permitted by statutory regulation or exceeds the permitted use, you will need to obtain permission directly from the copyright holder. To view a copy of this licence, visit http://creativecommons.org/licen ses/by/4.0/.

\section{References}

1. Cottle, R.W., Pang, J.S., Stone, R.E.: The linear complementarity problem. Academic Press, New York (1992)

2. Ferris, M.C., Pang, J.S.: Complementarity and variational problems. Philadephia, Pennsylvania (1997)

3. Lemke, C.E.: Bimatrix equilibrium points and mathematical programming. Manag. Sci. 11(7), 681-689 (1965)

4. Joaquim, J.J., Fernanda, M.P.: A block principal pivoting algorithm for larger-scale structure linear complementarity problems. Comput. Oper. Res. 5(21), 581-596 (1994)

5. Murty, K.G.: Linear Complement. Linear and Nonlinear Programming, Heldermann (1988)

6. Shi, X.J., Yang, L., Huang, Z.H.: A fixed point method for the linear complementarity problem arising from American option pricing. Acta Math. Appl. Sin. 4(32), 921-932 (2016)

7. Bai, Z.Z.: Modulus-based matrix splitting iteration methods for linear complementarity problems. Numer. Linear Algebra Appl. 17(6), 917-933 (2010)

8. Ahn, B.H.: Iterative methods for linear complementarity problems with upperbounds on primary variables. Math. Program. 26(3), 295-315 (1983)

9. Murty, K.G.: On the number of sulutions to the complementarity problem and spanning properties of complementary cones. Linear Algebra Appl. 5, 65-108 (1972)

10. Li, W.: A general modulus-based matrix splitting method for linear complementarity problems of $H$-matrices. Appl. Math. Lett. 26(12), 1159-1164 (2013)

11. Hadjidimos, A., Zhang, L.L.: Comparison of three classes of algorithms for the solution of the linear complementarity problem with an H+-matrix. J. Comput. Appl. Math. 336, 175-191 (2018)

12. Cottle, Richard W.: The principal pivoting method revisited. Math. Program. 48(1-3), 369-385 (1990)

13. Zheng, H., Li, W., Qu, W.: A non-modulus linear method for solving the linear complementarity problem. Linear Algebra Appl. 495, 38-50 (2016)

14. Bai, Z.Z., Evans, D.J.: Chaotic iterative methods for the linear complementarity problems. J. Comput. Appl. Math. 96(2), 127-138 (1998)

15. Chandrasekarn, R.: A special case of the complementary pivot problem. Oper. Res. 7, 263-268 (1970)

16. Cottle, Richard W., Sacher, Richard S.: On the solution of large, structured linear complementarity problems: the tridiagonal case. Math. Optim. 3(4), 321-340 (1976)

17. Dong, J.L., Jiang, M.Q.: A modified modulus method for symmetric positive-definite linear complementarity problems. Numer. Linear Algebra Appl. 16(2), 129-143 (2010) 
18. Bai, Z.Z., Dong, J.L.: A modified damped Newton method for linear complementarity problems. Numer. Algorithms 42(3-4), 207-228 (2006)

19. Graves, R.L.: A principal pivoting simplex algorithm for linear and quadratic programming. Oper. Res. 15(3), 482-494 (1967)

20. Hadjidimos, A., Tzoumas, M.: Nonstationary extrapolated modulus algorithms for the solution of the linear complementarity problem. Linear Algebra Appl. 431(1), 197-210 (2009)

21. Hadjidimos, A., Tzoumas, M.: The solution of the linear complementarity problem by the matrix analogue of the accelerated overrelaxation iterative method. Numer. Algorithms. 73(3), 665-684 (2016)

22. Jiang, Y.J., Zeng, J.P.: Direct algorithm for the solution of two-sided obstacle problems with M-matrix. Numer. Linear Algebra Appl. 18, 167-173 (2011)

23. Wu, S.L., Li, C.X.: Two-sweep modulus-based matrix splitting iteration methods for linear complementarity problems. J. Comput. Appl. Math. 302, 327-339 (2016)

24. Zhou, S.Z.: A direct method for the linear complementarity problem. J. Comput. Math. 8(2), $178-182(1990)$

25. Zhang, L., Hu, X.Y.: On the direct method for linear complementarity problem. Math. Numer. Sin. 16(1), 59-64 (1994)

26. Zeng, J.P., Jiang, Y.J.: Direct algorithms to solve the two-sided obstacle problem for an $M$ -matrix. Numer. Linear Algebra Appl. 13(7), 543-551 (2006)

27. Chen, X.J., Xiang, S.H.: Computation of error bounds for $P$-matrix linear complementarity problems. Math. Program. 106(3), 513-525 (2006)

28. Mathis, Roy, J. S., Pang: Error bounds for the linear complementarity problem with a $P$-matrix. Linear Algebra Appl. 132, 123-136 (1990)

29. Chen, X., Xiang, S.: Perturbation bounds of $P$-matrix linear complementarity problems. SIAM J. Optim. 18(4), 1250-1265 (2007)

30. Garcia-Esnaola, M., Pena, J.M.: A comparison of error bounds for linear complementarity problems of $H$-matrices. Linear Algebra Appl. 433(5), 956-964 (2010) 